\title{
Vascular morphology alterations during liver cirrhogenesis in rats
}

Authors: Geert Peeters ${ }^{1}$, Charlotte Debbaut ${ }^{1}$, Winnok H. De Vos ${ }^{2,3}$, Pieter Cornillie ${ }^{4}$, Thomas De Schryver ${ }^{5}$, Diethard Monbaliu ${ }^{6,7}$, Wim Laleman ${ }^{8,9,10}$, Patrick Segers ${ }^{1}$

${ }^{1}$ IBiTech - bioMMeda, Dept. Electronics and Information Systems, Ghent University, Belgium

${ }^{2}$ Lab. Cell Biology and Histology, Dept. Veterinary Sciences, University of Antwerp, Belgium

${ }^{3}$ Cell Systems and Imaging, Dept. Molecular Biotechnology, University of Ghent, Belgium

${ }^{4}$ Dept. Morphology, Faculty of Veterinary Medicine, Ghent University, Belgium

${ }^{5}$ Centre for X-Ray Tomography, Dept. Physics and Astronomy, Ghent University, Belgium

${ }^{6}$ Abdominal Transplant Surgery, University Hospitals Leuven, Belgium

${ }^{7}$ Dept. Microbiology and Immunology, KU Leuven, Belgium

${ }^{8}$ Gastroenterology \& Hepatology, University Hospitals Leuven, Belgium

${ }^{9}$ Jessa Hospital, Hasselt, Belgium

${ }^{10}$ Dept. Clinical and Experimental Medicine, KU Leuven, Belgium

\section{BACKGROUND AND AIMS}

To date, little is known about the hemodynamic consequences caused by liver cirrhosis, especially at the microlevel. To gain more insight in the vascular deterioration during cirrhogenesis, accurate 3D reconstructions of the hepatic circulation are a necessity. We have optimized two complementary techniques to acquire detailed 3D geometrical data of the rat liver circulation, covering the entire length scale of the hepatic vasculature, and applied it to an established rat model of cirrhosis.

\section{METHODS}

Cirrhosis was induced in rats according to the thioacetamide (TAA) protocol [1]. Prolonged TAA intoxication induces centrilobular necrosis and eventually homogenous macronodular cirrhosis after 18 weeks. Rats $(n=36)$ were sacrificed at $0,6,12$ and 18 weeks. At each time point, 5 rats were assigned to the vascular corrosion casting (VCC) technique and 4 rats to immunohistochemistry (IHC).

VCC entails injecting the casting resin PU4ii in the portal vein and hepatic artery. Lipiodol was added to the arterial mixture as a contrast agent to ensure a clear distinction between the vascular trees after high resolution micro-CT-scanning. The resulting datasets enabled reconstructing detailed 3D geometries of the hepatic macro- and microcirculation.

The IHC method includes staining $350 \mu \mathrm{m}$ thick liver slices with a generic endothelial marker antibody (RECA). To increase the liver slices' transparency and microscopic penetration depth, an adapted version of the clearing protocol CUBIC was applied [2]. Image stacks were subsequently recorded with a confocal microscope, and automatically segmented and 3D analysed using in-house developed software (DeLiver) allowing quantification of the microcirculation.

\section{RESULTS}

We were able to analyse and compare morphological parameters (radius, tortuosity, length, etc.) during cirrhogenesis. Our IHC results suggest that microcirculatory alterations deteriorate hepatic perfusion as the porosity (i.e. the number of sinusoids per unit of volume) steadily declines from 20.8 $\pm 2.3 \%$ (normal) to $11.4 \pm 3.1 \%$ (cirrhosis), and the mean sinusoidal radius significantly decreases $(p<0.05)$ for cirrhosis $(3.9 \pm 0.4 \mu \mathrm{m})$ compared to normal liver tissue $(4.4 \pm 0.3 \mu \mathrm{m})$. 


\section{CONCLUSION}

Our methodology allows examining the impact of liver cirrhosis on the morphology of the hepatic vasculature at different scales.

1. Laleman, $W$, et al. "A stable model of cirrhotic portal hypertension in the rat: thioacetamide revisited." EJCI 36.4 (2006): 242-249.

2. Susaki, E.A., et al., "Whole-brain imaging with single-cell resolution using chemical cocktails and computational analysis." Cell, 157.3 (2014): 726-39. 